\title{
Distinct Response of Fat and Gastrointestinal Tissue to Glucose in Gestational Diabetes Mellitus and Polycystic Ovary Syndrome
}

\author{
D. VEJRAZKOVA ${ }^{1}$, O. LISCHKOVA ${ }^{1}$, M. VANKOVA ${ }^{1}$, S. STANICKA $^{1}$, J. VRBIKOVA $^{1}$, \\ P. LUKASOVA ${ }^{1}$, J. VCELAK ${ }^{1}$, G. VACINOVA ${ }^{1}$, B. BENDLOVA ${ }^{1}$ \\ ${ }^{1}$ Department of Molecular Endocrinology, Institute of Endocrinology, Prague, Czech Republic
}

Received April 26, 2016

Accepted September 16, 2016

On-line December 16, 2016

\begin{abstract}
Summary
Gestational diabetes mellitus (GDM) and polycystic ovary syndrome (PCOS) are distinct pathologies with impaired insulin sensitivity as a common feature. The aim of this study was to evaluate the response of fat tissue adipokines and gastrointestinal incretins to glucose load in patients diagnosed with one of the two disorders and to compare it with healthy controls. Oral glucose tolerance test (oGTT) was performed in 77 lean young women: 22 had positive history of GDM, 19 were PCOS patients, and 36 were healthy controls. Hormones were evaluated in fasting and in $60 \mathrm{~min}$ intervals during the $3 \mathrm{~h}$ oGTT using Bio-Plex ProHuman Diabetes 10-Plex Assay for C-peptide, ghrelin, GIP, GLP1, glucagon, insulin, leptin, total PAI1, resistin, visfatin and Bio-Plex ProHuman Diabetes Adipsin and Adiponectin Assays (Bio-Rad). Despite lean body composition, both PCOS and GDM women were more insulin resistant than controls. Significant postchallenge differences between the GDM and PCOS groups were observed in secretion of adipsin, leptin, glucagon, visfatin, ghrelin, GIP, and also GLP1 with higher levels in GDM. Conversely, PCOS was associated with the highest resistin, C-peptide, and PAI1 levels. Our data suggest that decreased insulin sensitivity observed in lean women with GDM and PCOS is associated with distinct hormonal response of fat and gastrointestinal tissue to glucose load.
\end{abstract}

\section{Key words}

Gestational diabetes mellitus - Polycystic ovary syndrome • Glucose tolerance • Adipokines • Incretins

\section{Corresponding author}

D. Vejrazkova, Department of Molecular Endocrinology, Institute of Endocrinology, Narodni 8, 11694 Prague 1, Czech Republic. Fax: +420224905 325. E-mail: dvejrazkova@endo.cz

\section{Introduction}

Gestational diabetes mellitus (GDM) and polycystic ovary syndrome (PCOS) are pathologies with one common denominator - impaired insulin sensitivity (IS) (Wei et al. 2014, Ravn 2015). This unfavorable health condition, characterized by reduced ability of insulin to exert its biological effect on target tissues, can be caused by an impairment of wide range of physiological regulations. Insulin resistance is a consequence of disturbed glucose and lipid metabolism, in which impaired energy homeostasis, excess and/or impaired function of adipose tissue, gastrointestinal hormonal dysfunction, altered gut microbiome, chronic inflammation, and external factors like diet, physical activity, stress, environment, or even conditions of prenatal development and final birth weight may play a role. In this complex and genetically strongly influenced process are involved also hormonal active substances known as adipokines and incretins.

Adipokines such as leptin, adiponectin, resistin, visfatin or adipsin are secreted by white adipose tissue, which is now recognized to be an active participant in glucose homeostasis. Recently, this evidence has become robust suggesting that obesity and inflammation are major components of insulin resistance. One of the mechanisms described in patients with metabolic 
syndrome characterized by excess visceral adipose tissue is that long-term exposure to higher adipokine levels leads to a chronic subinflammatory state that is involved in development of insulin resistance (Thomas et al. 2015). In most individuals, insulin resistance and obesity coexist. However, also lean subjects can develop inflammation-associated insulin resistance (Mehta et al. 2010). Lean insulin resistant subjects may have even higher pro-inflammatory adipokine profile than overweight but insulin sensitive subjects (Moscavitch et al. 2016). This implies that the degree of adipose tissue inflammation, not obesity per se, is a precondition for the development of insulin resistance (Hamada et al. 2011).

Incretins such as glucagon-like peptide 1 (GLP1) or glucose-dependent insulinotropic polypeptide (GIP) are gut hormones secreted from the enteroendocrine cells into the blood after eating. Their main physiological function is to potentiate glucose-stimulated insulin secretion in a glucose-dependent way. Therefore, there has been a lot of interest in developing incretin-based therapy for type 2 diabetes mellitus.

Ghrelin is a peptide produced by gastrointestinal tract. It regulates hunger and energy distribution through signaling in the central nervous system. More specifically, ghrelin is hypothesized to stimulate GLP1 secretory response to ingested nutrients and is discussed in connection with a new incretin enhancer therapy approach (DeMarco and Sowers 2015).

PAI1 (plasminogen activator inhibitor 1) is a major regulator of the fibrinolytic system. It is produced by the endothelium, but is also secreted by other tissue types, such as adipose tissue, liver, lung, and muscle (Binder et al. 2002). Increased PAI1 levels in plasma accompany symptoms of metabolic syndrome, such as glucose intolerance and insulin resistance. Under some pathological conditions like sepsis or other acute and chronic inflammatory diseases including atherosclerosis, endothelial cells secrete a large amount of PAI1 in response to inflammatory cytokines (Bouchard et al. 2010).

Despite large body of data that is shaping our understanding of mechanisms underlying insulin resistance development, this process is exceptionally complex and the data can be difficult to reconcile. As health organizations throughout the world struggle to find solutions to this largely preventable health issue, it is highly desirable to offer new insights and potential avenues for preventive intervention. The aim of this study was to monitor the response of the above stated fat tissue adipokines and gastrointestinal incretins to glucose load and to compare it between patients suffering from one of the two distinct metabolic disorders associated with insulin resistance - GDM and PCOS - in order to uncover possible differences in mechanism underlying deterioration of IS in these women. All the observations were evaluated in relation to healthy control women with normal glucose tolerance.

\section{Methods}

The $3 \mathrm{~h}$ oral glucose tolerance test (oGTT) with $75 \mathrm{~g}$ of glucose was performed in 77 lean women: 22 women with a history of GDM (BMI range $18.5-24.9 \mathrm{~kg} / \mathrm{m}^{2}$, age $34.9 \pm 4.62$ years) diagnosed by the criteria based on WHO guidelines and the Czech Gynecological and Obstetrical Society meeting the 0.5-1 year interval after delivery, all non breastfeeding, 19 PCOS patients (BMI range 18.7-24.8 $\mathrm{kg} / \mathrm{m}^{2}$, age 26.7 \pm 5.41 years) diagnosed according to the ESHRE2004 consensus, and 36 healthy controls with a regular menstrual cycle and normal glucose tolerance (glycemia in $120 \mathrm{~min}$ of $\mathrm{oGTT}<7.8 \mathrm{mmol} / \mathrm{l}$, BMI range $18.8-24.9 \mathrm{~kg} / \mathrm{m}^{2}$, age $30.4 \pm 9.67$ years). These groups did not differ in mean BMI, WHR, or body fat content. Details regarding characterization of the tested groups are listed in Table 1.

Body weight, height, waist, hip, and abdominal circumferences were measured in order to calculate body mass index (BMI), body adiposity index (BAI) and to evaluate body fat distribution by means of waist circumference and waist to hip ratio (WHR).

All 12 analyzed hormones evaluated in the fasting state and then in 60 min intervals during the $3 \mathrm{~h}$ oGTT were determined in plasma using Bio-Plex ProHuman Diabetes 10-Plex Assay, Bio-Rad (C-peptide, ghrelin, GIP, GLP1, glucagon, insulin, leptin, total PAI1, resistin, visfatin) and Bio-Plex ProHuman Diabetes Adipsin and Adiponectin Assays, Bio-Rad (adipsin, adiponectin). For these assays, blood samples were collected into glass tubes with $\mathrm{K}_{3}$ EDTA $1.5 \mathrm{mg} / \mathrm{ml}$ and Aprotinin $250 \mathrm{KIU} \quad$ (BD Vacutainer ${ }^{\circledR}$, New Jersey, USA), treated with DPP IV Protease Inhibitor Coctail (Sigma-Aldrich, St. Louis, USA), and stored at $-80^{\circ} \mathrm{C}$ until processed. For methodological problems encountered in the determination of glucagon by Bio-Plex, RIA method (Euria-glucagon EuroDiagnostica, Malmö, Sweden) was preferred to evaluate basal and postchallenge glucagon plasma concentrations. 
Table 1. Characteristics of the groups.

\begin{tabular}{|c|c|c|c|c|}
\hline & GDM & PCOS & Controls & \multirow{2}{*}{$\begin{array}{c}\text { Age-adjusted } \\
\text { ANCOVA } \\
\text { p-level }\end{array}$} \\
\hline & $\mathrm{n}=\mathbf{2 2}$ & $n=19$ & $\mathrm{n}=36$ & \\
\hline Age (years) & $34.9(32.8 ; 37.0)$ & $26.7(24.8 ; 28.8)$ & $30.4(28.9 ; 32.0)$ & - \\
\hline$B M I\left(\mathrm{~kg} / \mathrm{m}^{2}\right)$ & $22.0(21.5 ; 22.6)$ & $21.8(21.2 ; 22.4)$ & $21.6(21.1 ; 22.0)$ & 0.59 \\
\hline$W H R$ & $0.76(0.75 ; 0.77)$ & $0.73(0.72 ; 0.75)$ & $0.75(0.74 ; 0.76)$ & 0.28 \\
\hline Waist $(\mathrm{cm})$ & $74.0(72.3 ; 75.9)$ & $70.7(69.1 ; 72.4)$ & $71.6(70.3 ; 72.9)$ & 0.30 \\
\hline Abdomen $(\mathrm{cm})$ & $84.2(82.4 ; 86.1)$ & $80.1(78.5 ; 81.9)$ & $80.5(79.3 ; 81.9)$ & 0.08 \\
\hline$B A I(\%)$ & $27.1(26.3 ; 27.9)$ & $26.4(25.6 ; 27.3)$ & $26.2(25.6 ; 26.9)$ & 0.65 \\
\hline BP systolic ( $\mathrm{mm} \mathrm{Hg})$ & $108(104 ; 112)$ & $102(99 ; 107)$ & $108(105 ; 112)$ & 0.20 \\
\hline BP diastolic ( $\mathrm{mm} \mathrm{Hg})$ & $66(64 ; 68)$ & $64(62 ; 67)$ & $68(66 ; 70)$ & 0.24 \\
\hline Basal glycemia (mmol/l) & $4.74(4.62 ; 4.87)$ & $4.52(4.39 ; 4.65)$ & $4.54(4.45 ; 4.64)$ & 0.31 \\
\hline$H O M A-I R$ & $1.14(1.01 ; 1.31)$ & $1.11(0.98 ; 1.28)$ & $0.83(0.76 ; 0.91)$ & $0.004 *$ \\
\hline$H O M A-F$ & $87.7(74.8 ; 103.5)$ & $112.4(93.9 ; 136.4)$ & $83.6(74.0 ; 94.9)$ & 0.23 \\
\hline IS-Matsuda index & $7.4(6.5 ; 8.3)$ & $7.4(6.4 ; 8.1)$ & $10.2(9.5 ; 10.2)$ & $<0.001 *$ \\
\hline IS-Cederholm index & $59.5(55.8 ; 63.4)$ & $63.1(59.2 ; 67.3)$ & $72.4(68.9 ; 76.2)$ & $0.004^{*}$ \\
\hline Insulinogenic index (IU/mol) & $12.0(10.0 ; 14.6)$ & $18.1(14.6 ; 23.0)$ & $14.5(12.5 ; 16.9)$ & 0.82 \\
\hline Total cholesterol (mmol/l) & $4.8(4.5 ; 5.0)$ & $4.4(4.2 ; 4.6)$ & $4.3(4.2 ; 4.5)$ & 0.16 \\
\hline HDL cholesterol (mmol/l) & $1.58(1.49 ; 1.68)$ & $1.61(1.51 ; 1.72)$ & $1.59(1.51 ; 1.67)$ & 0.21 \\
\hline LDL cholesterol (mmol/l) & $2.7(2.5 ; 2.9)$ & $2.3(2.1 ; 2.5)$ & $2.3(2.1 ; 2.4)$ & 0.14 \\
\hline$T A G(\mathrm{mmol} / \mathrm{l})$ & $0.89(0.80 ; 0.99)$ & $0.88(0.78 ; 0.98)$ & $0.78(0.71 ; 0.84)$ & 0.28 \\
\hline
\end{tabular}

* The difference between the GDM group and Controls and concurrently between PCOS group and Controls is significant. Values are given as mean ( $95 \%$ confidence interval).

To assess insulin sensitivity (IS) and beta cell function, four indices were calculated. Two of them are based on fasting glucose and insulin levels: homeostasis model of insulin resistance HOMA-IR $=$ insulin $_{0 \min } \mathrm{X}$ glucose $_{0 \min } / 22.5$ and homeostasis model of beta cell function $\mathrm{HOMA}-\mathrm{F}=20 \mathrm{x}$ insulin $_{0 \min } /\left(\right.$ glucose $\left._{0 \min }-3.5\right)$. Two indices of IS are based on stimulated glycemia and insulinemia levels: Matsuda index $=10^{4} / \sqrt{ }$ (mean

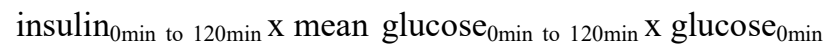
$\mathrm{x}$ insulin $\left._{0 \mathrm{~min}}\right)$ and Cederholm index $=[75000+$ (glucose $_{0 \min }-$ glucose $\left._{120 \mathrm{~min}}\right) \times 39.33 \mathrm{x}$ body weight] / (120 $\mathrm{x}$ mean glucose $\mathrm{g}_{0 \min }$ to $120 \mathrm{~min} \mathrm{x} \log$ mean insulin ${ }_{0 \min }$ to $\left.120 \mathrm{~min}\right)$. Insulinogenic index calculated as insulin $_{30 \min }-$ insulin $\left._{0 \min }\right) /\left(\right.$ glucose $_{30 \mathrm{~min}}-$ glucose $\left._{0 \mathrm{~min}}\right)$ was used to assess early insulin response during the first $30 \mathrm{~min}$ of the test. For these calculations, serum glucose by enzymatic reference method with hexokinase (Cobas 6000, Roche Diagnostics, Indianapolis, USA) and insulin by ECLIA (Cobas 6000, Roche Diagnostics) were measured.

Lipid profile was assessed by total cholesterol (enzymatic colorimetric test; Cobas 6000, Roche
Diagnostics), high density lipoprotein (HDL) (homogeneous enzymatic colorimetric test; Cobas 6000, Roche Diagnostics), low density lipoprotein (LDL) (homogeneous enzymatic colorimetric test; Cobas 6000, Roche Diagnostics), and triacylglycerol (TAG) concentrations (enzymatic colorimetric test; Cobas 6000, Roche Diagnostics).

Complete records of standardized questionnaires monitoring anamnestic data and life-style information including self-reported quality of sleep were collected from all our participants.

\section{Statistics}

Considering the skewed distribution and nonconstant variance in most of the evaluated variables, these were transformed by power transformation to data symmetry and homoscedasticity prior further processing. The homogeneity and distribution of the transformed data was checked by residual analysis. Then parametric Analysis of Covariance (Statgraphics Centurion XVI 16.0.07 software, Statpoint Technologies, Inc., Virginia, 
USA) was used for comparing anthropometric and basic biochemical characteristics between the groups in Table 1. Multiple Comparisons General Linear Model (GLM) Anova with "group" and "time" during the oGTT as independent categorical factors was applied to monitor adipokine and incretin levels in the three analyzed groups. Adjustment for age was applied. Bonferroni All-pairwise Multiple Comparison Test was used to identify the two significantly different groups among the three tested. The p-values $<0.05$ (two tailed) were considered to be significant.

The study protocol was in accordance with institutional ethic guidelines and the national laws and all subjects gave their written informed consent to participate in the study.

\section{Results}

In spite of similar body composition (almost identical BMI and body adiposity index, Table 1), PCOS and GDM groups were more insulin resistant than controls as is apparent from lower Matsuda and Cederholm indices of insulin sensitivity and higher HOMA-IR index of insulin resistance (Table 1). The postchallenge glycemia was higher in GDM group compared to controls and PCOS women with maximal difference in $60^{\text {th }}$ min of the oGTT (Fig. 1).

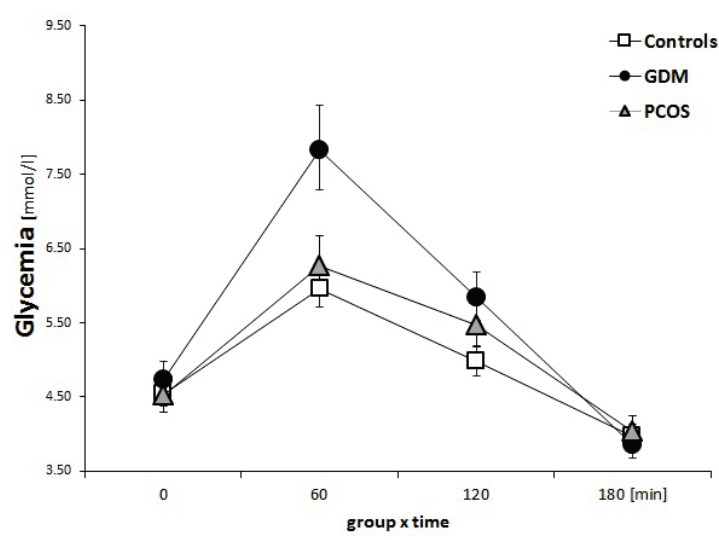

Factor"group": $F=8.4, p<0.001$; Factor"time": $F=110.1, p<0.001$; Interaction"group $x$ time": $F=3.3, p=0.004$ GDM - Controls *

GDM - PCOS

Controls - PCOS

*denotes a significant difference

Fig. 1. Serum levels of glycemia during the $3 \mathrm{~h}$ oGTT in GDM, PCOS, and control women. General Linear Model with "group" and "time" as independent categorical factors, age-adjusted data.
Concerning the glucose-induced changes in concentrations of the 12 analyzed hormones, Figures 2, 3, and 4 illustrate postchallenge differences in their response between the groups (Factor "group" in the legend of Figs 2, 3 and 4) at a specific time of the oGTT (Factor "time" in the legend of Figs 2, 3 and 4) as well as interactions between the two factors (Interaction "group $\mathrm{x}$ time" in the legend of Figs 2, 3 and 4). Calculations are adjusted for age.

Adipsin, visfatin, ghrelin, and GLP1 showed the highest levels in GDM group and the lowest in PCOS patients. These highly significant differences persisted in all four assessed hormones throughout the whole test. Leptin and glucagon concentrations were also the highest in GDM women, but the lowest leptinemia was observed in controls. Glucagonemia did not differ between controls and PCOS patients. Concentrations of resistin, C-peptide, and PAI1 were the highest in PCOS patients. Adiponectin was the only hormone from all analyzed in the study with the highest levels in controls.

Monitoring and comparing the shape of the oGTT curve between the groups, i.e. evaluation of differences in hormone response to glucose separately in each of the four times during the test, revealed that most of the hormones showed similar response direction after glucose administration across the groups. This is statistically processed as interaction between the factor "group" and the factor "time" in Figures 2, 3 and 4. C-peptide decreased more steeply in controls compared to GDM and PCOS during the $2^{\text {nd }}$ hour (borderline significance of $\mathrm{p}=0.04$ for interaction "group $\mathrm{x}$ time", Fig. 3).

As regards beta cell function, peripheral insulin levels were lower in controls compared to GDM and PCOS women, while peripheral C-peptide was significantly higher in PCOS compared with GDM group and with controls (Fig. 3). No difference between the groups was found in early insulin response expressed by insulinogenic index (Table 1).

Furthermore, our results indicate that hormonal response can be distinct or even opposit during the $2^{\text {nd }}$ hour in comparison with the $3^{\text {rd }}$ hour of the oGTT. This is remarkable especially in adipsin, glucagon, ghrelin, and, among GDM women, also in resistin and leptin (Figs 2, 3 and 4). 


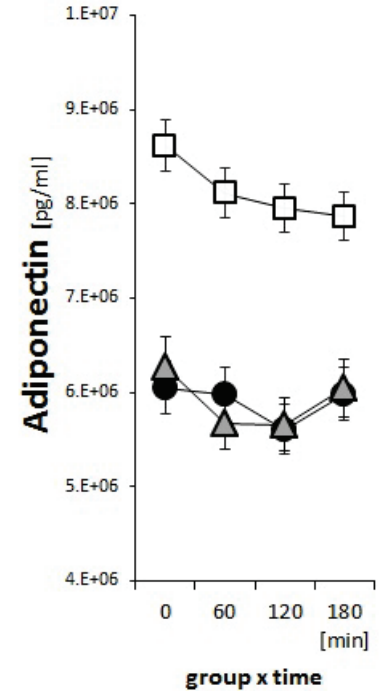

Factor "group": F=148.3, p<0.001 GDM - Controls GDM - PCOS

Controls - PCOS *

Factor"time": $F=4.3, p=0.006$

* denotes a significant

Fig. 2. Plasma levels of adipokines by multiplex assays during the $3 \mathrm{~h}$ oGTT in GDM, PCOS, and control women. General Linear Model with "group" and "time" as independent categorical factors, age-adjusted data.
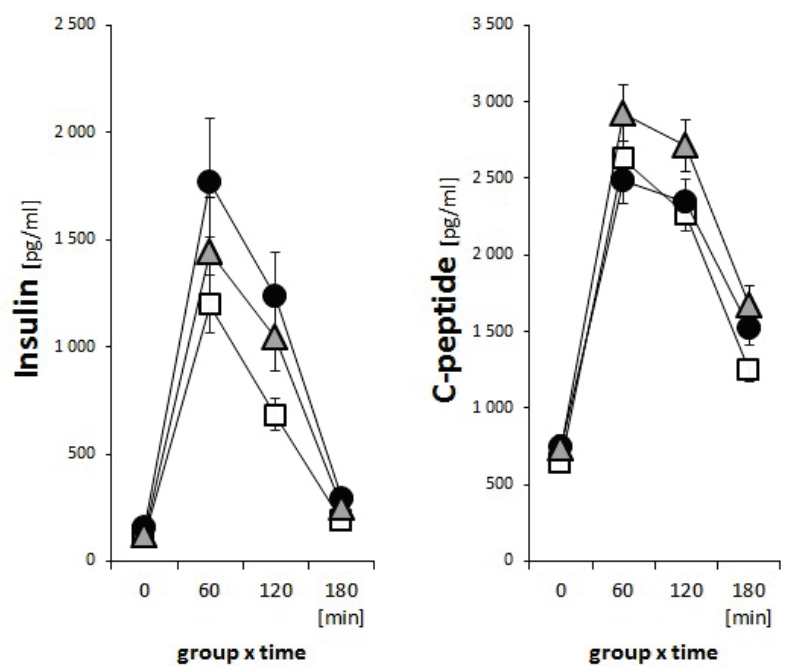

Factor "group": $\mathrm{F}=11.3, \mathrm{p}<0.001$

GDM - Controls *

GDM - PCOS

Controls-PCOS *

group $x$ time

Factor "group": $\mathrm{F}=12.1, \mathrm{p}<0.001$

GDM - Controls

GDM - PCOS

Controls - PCOS *

Factor"time": F=468.1, p<0.001

Interaction"group $x$ time" $F=1.0, p=0.43$

* denotes a significant difference

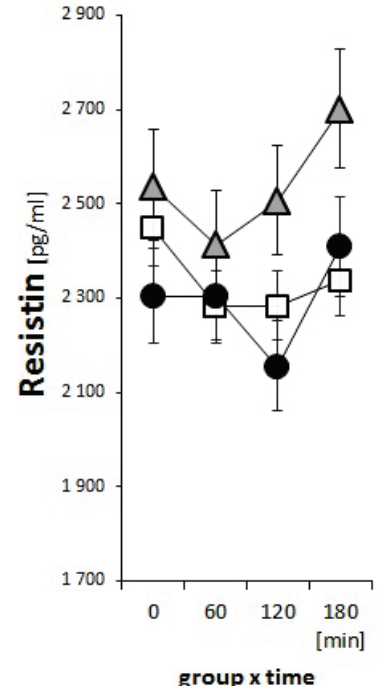

Factor "group": $\mathrm{F}=8.6, \mathrm{p}<0.001$

GDM - Controls

GDM - PCOS *

Controls - PCOS *

Factor"time": $\mathrm{F}=4.2, \mathrm{p}=0.007$

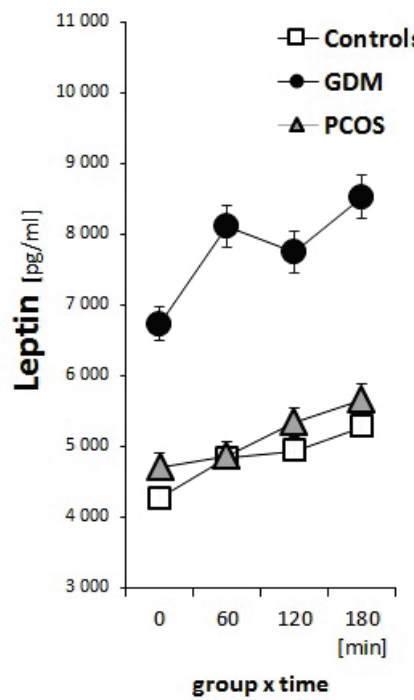

Factor "group": F=332.9, p<0.001

GDM - Controls *

GDM - PCOS *

Controls - PCOS *

Factor"time": $F=39.0, p<0.001$

22 Interaction"group $\mathrm{x}$ time" $\mathrm{F}=1.9, \mathrm{p}=0.081$

Fig. 3. Plasma levels of glucose metabolism regulators by multiplex assays during the $3 \mathrm{~h}$ oGTT in GDM, PCOS, and control women. General Linear Model with "group" and "time" as independent categorical factors, age-adjusted data. 


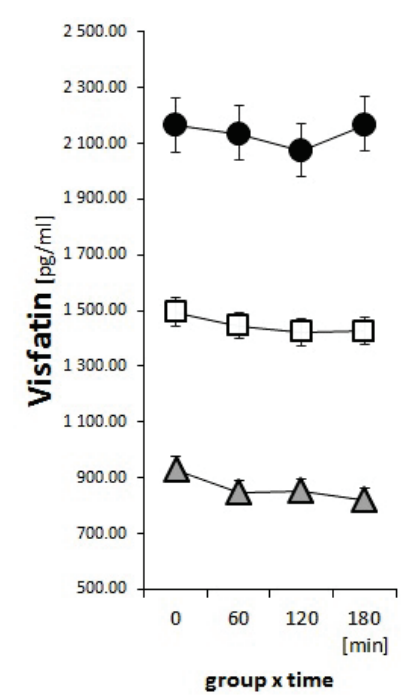

Factor "group": $F=530.9, p<0.001$ GDM - Controls * GDM - PCOS *

Controls-PCOS *

Factor"time": $\mathrm{F}=2.6, \mathrm{p}=0.055$

Interaction"group $\mathrm{x}$ time" $\mathrm{F}=0.7, \mathrm{p}=0.65$ * denotes a significant difference

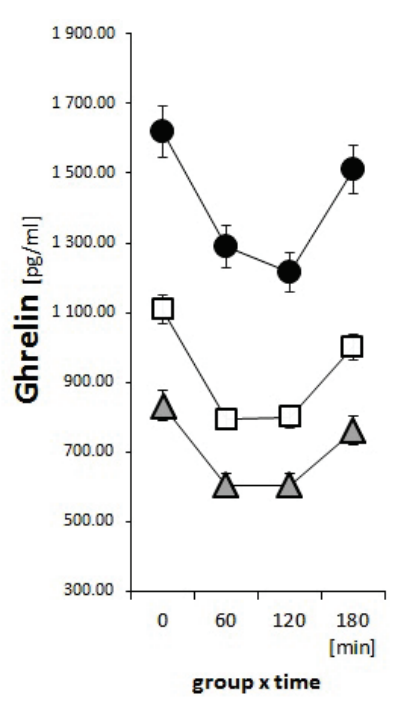

Factor "group": F=286.5, p<0.001

GDM - Controls * GDM - PCOS *

Controls-PCOS *

Factor"time": $F=71.2, p<0.001$

Interaction"group $\mathrm{x}$ time" $\mathrm{F}=0.5, \mathrm{p}=0.82$

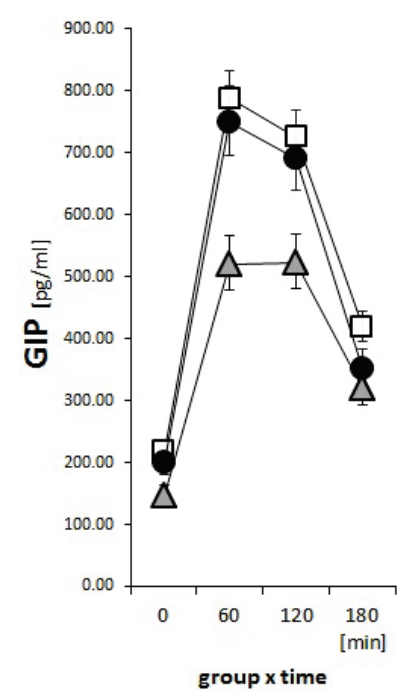

Factor "group": F=33.4, p<0.001

GDM - Controls *

GDM - PCOS *

Controls-PCOS *

Factor"time": $F=370.8, p<0.001$

Interaction"group $\mathrm{x}$ time" $\mathrm{F}=1.3, \mathrm{p}=0.28$

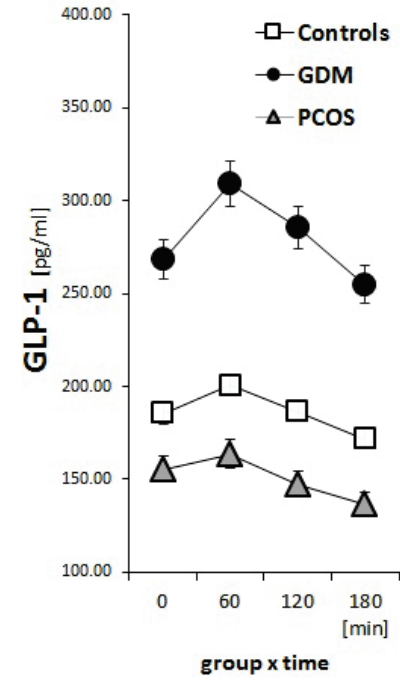

Factor "group": $F=332.4, p<0.001$

GDM - Controls *

GDM - PCOS *

Controls - PCOS *

Factor"time": $F=23.2, p<0.001$

Interaction"group $\mathrm{x}$ time" $\mathrm{F}=0.9, \mathrm{p}=0.49$

Fig. 4. Plasma levels of incretins and adipokines by multiplex assays during the $3 \mathrm{~h}$ oGTT in GDM, PCOS, and control women. General Linear Model with "group" and "time" as independent categorical factors, age-adjusted data.

\section{Discussion}

Decreased IS, observed in lean young women with positive history of GDM and in lean women suffering from PCOS compared with control normal-weight women in our study, is associated with markedly divergent hormonal response of fat and gastrointestinal tissue to glucose load. To our best knowledge, such comparative specification of adipokine and incretin-based pathophysiological pathways which take part in underlying insulin resistance in the two distinct endocrine diseases, PCOS and GDM, has not been published before.

The most obvious and consistent differences between GDM and PCOS women are in secretion of adipsin, leptin, glucagon, visfatin, ghrelin, GIP, and also in GLP1 with significantly higher levels in GDM group. Conversely, PCOS was associated with the highest resistin, C-peptide, and PAI1 levels.

Adipsin is expressed and secreted at high levels by adipose tissue. It stimulates glucose transport and adipocyte triglyceride synthesis through an insulindependent mechanism (Yasruel et al. 1991, Maslowska et al. 1997). Adipsin is positively correlated with BMI (Chedraui et al. 2014), but obese type 2 diabetics with beta cell failure are deficient in adipsin (Lo et al. 2014). These findings identify adipsin as a circulating factor linking fat cells to beta cell function, more specifically, adipsin potentiates insulin secretion (Lo et al. 2014). Our observation of higher adipsin levels in lean women with a positive GDM history, whose postchallenge glucose levels were higher compared to PCOS and control group, may reflect adipsin-mediated support of insulin secretion to restore normoglycemia after glucose consumption.

Leptin has been acknowledged as a major adipocyte-derived endocrine signal in the homeostatic control of body weight. Subcutaneous fat content is a major determinant of circulating leptin levels. Leptin inhibits appetite, stimulates thermogenesis, decreases glucose, and reduces body weight and fat mass (Yadav et al. 2013). Beyond its metabolic functions, leptin is a pleiotropic cytokine involved in inflammation (Fantuzzi and Faggioni 2000). High leptinemia observed in our GDM group with the highest postchallenge glycemia, insulinemia, HOMA-IR, and the lowest Cederholm index of IS is consistent with a positive correlation of leptin with insulin resistance described in other studies (Nasrat et al. 2016, Osegbe et al. 2016). In PCOS women, fasting and postchallenge $(120 \mathrm{~min})$ levels of leptin were also higher compared to controls. Some studies have previously reported increased circulating leptin concentrations in PCOS independently of insulin resistance and suggested that leptin had a role in its 
pathogenesis (Sepilian et al. 2006, Gregoraszczuk and Rak 2015, Rizk and Sharif 2015). Higher leptinemia in our PCOS women thus could be attributed, along with lower IS in this group, also to the differences in steroid spectra specifying this syndrome.

Glucagon is a counterregulatory hormone that promotes hepatic glucose production, thereby preventing hypoglycemia in normal physiology. In healthy individuals, glucose load suppresses glucagon release. In diabetics, glucose does not suppress glucagon to the same extent (Kulina and Rayfield 2016). Hence, glucagon antagonizing agents are likely to be of value in the diabetes treatment. A longitudinal study showed that increased glucagon secretion was evident in patients who eventually developed impaired glucose tolerance even before the impaired glucose tolerance was diagnosed (Ahren 2009). In this respect, our observations of higher glucose-stimulated glucagon in women with positive GDM history are in accordance with their highest postchallenge glycemia and insulinemia. Finding of Ahren (2009) therefore refers to this group as at risk regarding the future development of glucose intolerance.

Visfatin, an adipokine preferentially expressed in visceral adipose tissue, exerts pro-inflammatory and immunomodulating properties and was described to be higher in obese subjects (Ahmed et al. 2015). Visfatin also has insulin-sensitizing and insulin-mimetic effect, so attention attracts its possible application in glycemic control. In our study, the highest visfatin levels were found in GDM positive women. Elevated plasma visfatin concentration has already been demonstrated in pregnant gestational diabetics (Lewandowski et al. 2007, Ferreira et al. 2011). Lewandowski et al. (2007) reported positive correlations of plasma visfatin with concentrations of both fasting and postchallenge insulinemia in GDM pregnant women. Ferreira et al. (2011) revealed an increased plasma visfatin level in pregnant women who subsequently developed GDM, suggesting that visfatin could be a potential biomarker for predicting GDM. Interestingly, data indicating inverse relationship between visfatin and sleep duration have been published (Hayes et al. 2011) and confirmed in direct relation to the sleep loss-associated impairment of postprandial glucose metabolism (Benedict et al. 2012). Therefore, it is likely that mild sleep deprivation indicated in questionnaires by our GDM women, who are all mothers meeting 0.5-1 year interval after delivery and caring for their babies also at night, plays an important role in our observation of higher visfatin and postchallenge glucose levels.
Ghrelin participates in regulation of nutrient sensing, food intake, and energy balance including glucose metabolism (DeMarco and Sowers 2015). It has orexigenic effect and is known as the "hunger hormone". In insulin resistant subjects, ghrelin concentrations were systematically lower than in healthy controls (Verhulst and Depoortere 2012). Also after an oral glucose administration, ghrelin levels were reported to be lower in subjects with more pronounced insulin resistance (Greenman et al. 2004). This is in agreement with our finding of lower ghrelin in PCOS group which is more insulin resistant than controls. Paradoxically, both fasting and stimulated ghrelin levels were the highest in GDM women showing the highest postchallenge glycemia, insulinemia, and the lowest Cederholm-derived IS. Again, disturbed sleep could be one possible explanation. Strong link between low-quality or mistimed sleep and changes in metabolic control including appetite-signaling hormones is well documented (Cedernaes 2015). Hence, in new mothers caring for their $0.5-1$ year old babies, ghrelin may be elevated in response to sleep restriction.

Also the gut-derived incretins GIP and GLP1, like stomach-derived ghrelin, are tightly connected to wholebody energy metabolism and meal consumption. While ghrelin concentrations are highest shortly before usual meal time, GIP and GLP1 are secreted after nutrient ingestion, which is seen from the responses of the three hormones (Fig. 4). As mentioned in the introduction, GIP and GLP1 work to augment glucose-stimulated insulin secretion. According to some studies, low incretin levels are indicative of glucose tolerance impairment (Rask et al. 2004, Zhang et al. 2012). These conclusions correspond well with our observation in PCOS women, but are conflicting in the GDM group. Nevertheless, Vollmer et al. (2008) found that deterioration in glucose homeostasis can develop in the absence of any disorder in GIP or GLP1 secretion. Owing to the fact that incretins have an important physiological function in augmenting postprandial insulin secretion, we can assume that higher incretin release observed in GDM women is an integral part of the process targeted at normalization of postchallenge glycemia, significantly higher in this group.

Different kind of hormonal reaction with less pronounced differences between the groups and characterized by the highest levels in PCOS patients was observed in resistin, C-peptide, and PAI1.

As stated in the introduction, secretion of PAI1 may be induced in response to inflammatory cytokines. In our study, higher PAIl levels were observed in PCOS 
patients. This correlates well with high pro-inflammatory resistin and low anti-inflammatory adiponectin. Nevertheless, other pro-inflammatory adipokines adipsin and visfatin were low in PCOS group. This inconsistent observation does not indicate that rise in PAI1 in our PCOS women is due to the action of inflammatory cytokines. More likely, our results confirm recent observation describing elevated levels of PAI1 as a novel independent biomarker and predictor of insulin resistance in normal-weight women with PCOS (Aziz et al. 2015, Cassar et al. 2015).

As mentioned above, the highest resistin levels were detected in PCOS women. Higher serum resistin has already been described among normal-weight PCOS patients in comparison with controls (Farshchian et al. 2014). Interestingly, resistin mRNA expression in adipocytes was found to be twice higher in PCOS patients implying resistin have a local paracrine mode of action in the PCOS pathogenesis (Seow et al. 2004, Seow et al. 2007).

Adiponectin was the only hormone with the highest levels in controls. Adiponectin is expressed in adipose tissue, but paradoxically correlates negatively with obesity. It plays a crucial role in the regulation of glucose metabolism and IS (Ghoshal et al. 2015). Epidemiological studies revealed that low serum adiponectin can be an excellent biomarker for predicting type 2 diabetes (Spranger et al. 2003). Finding of lower adiponectin in lean PCOS and GDM women in comparison with lean controls confirms adiponectin as adiposity-independent marker of decreased IS in these two groups.

Pancreatic beta cell function was evaluated by means of basal and postchallenge peripheral insulin and C-peptide concentrations. Lower stimulated insulinemia in controls compared to PCOS and GDM group reflects better IS in this group, which corresponds well with calculated IS indices (Table 1). The highest postchallenge peripheral insulin levels were observed in GDM women. However, postchallenge C-peptide levels were not as high in this group. Considering equimolar secretion of insulin and C-peptide into the circulation, this observation may be explained by lower hepatic insulin extraction in women with positive history of GDM.
Besides, it has been demonstrated by our study that hormonal response can be distinct or even opposite during the $2^{\text {nd }}$ hour of the oGTT and during the $3^{\text {rd }}$ hour of the prolonged $3 \mathrm{~h}$ oGTT. Most studies refer to the standard $2 \mathrm{~h}$ oGTT. That is why it is important to take the duration of the oGTT into account while evaluating the glucose effect to hormones like adipsin, resistin, glucagon, ghrelin, or leptin.

The merit of our approach, which is high somatometric, age, ethnic, and also diagnostic homogeneity of the study groups, brings also certain limitation, which is number of participants. This limitation shows where new efforts need to be made. Based on these results, the next step would be to build a stronger overall evidence base concerning the issue.

In conclusion, IS according to HOMA-IR, Matsuda, and Cederholm indices was lower in women with a history of GDM and in PCOS patients in comparison with controls of similar BMI. Evaluation of hormonal response to glucose load revealed significant postchallenge differences between the groups with the highest concentrations of adipsin, visfatin, ghrelin, and GLP1 in GDM group and the lowest in PCOS patients. Conversely, PCOS was associated with the highest resistin, C-peptide, and PAI1 levels. Our observation suggests that decreased IS identified in normal-weight women with GDM history and in lean PCOS patients is based on distinct signaling of adipose and gastrointestinal tissue. Thus, different mechanisms are relevant in the development of insulin resistance characterizing these two pathologies. This descriptive study gives added value to the understanding of the etiopathology of impaired IS in the two specific endocrine diseases. Deepening knowledge of the underlying mechanisms is of great importance for the development of new strategies for type 2 diabetes prevention and treatment.

\section{Conflict of Interest}

There is no conflict of interest.

\section{Acknowledgements}

This work was supported by the IGA MH CR NT135444/2012, MH CZ RVO EÚ 00023761.

\section{References}

AHMED MB, ISMAIL MI, MEKI AR: Relation of osteoprotegerin, visfatin and ghrelin to metabolic syndrome in type 2 diabetic patients. Int J Health Sci 9: 127-139, 2015.

AHREN B: Beta- and alpha-cell dysfunction in subjects developing impaired glucose tolerance: outcome of a 12-year prospective study in postmenopausal Caucasian women. Diabetes 58: 726-731, 2009. 
AZIZ M, SIDELMANN JJ, FABER J, WISSING ML, NAVER KV, MIKKELSEN AL, NILAS L, SKOUBY SO: Polycystic ovary syndrome: cardiovascular risk factors according to specific phenotypes. Acta Obstet Gynecol Scand 94: 1082-1089, 2015.

BENEDICT C, SHOSTAK A, LANGE T, BROOKS SJ, SCHIÖTH HB, SCHULTES B, BORN J, OSTER H, HALLSCHMID M: Diurnal rhythm of circulating nicotinamide phosphoribosyltransferase (Nampt/visfatin/PBEF): impact of sleep loss and relation to glucose metabolism. J Clin Endocrinol Metab 97: E218-E222, 2012.

BINDER BR, CHRIST G, GRUBER F, GRUBIC N, HUFNAGL P, KREBS M, MIHALY J, PRAGER GW: Plasminogen activator inhibitor 1: physiological and pathophysiological roles. News Physiol Sci 17: 56-61, 2002.

BOUCHARD L, VOHL MC, LEBEL S, HOULD FS, MARCEAU P, BERGERON J, PÉRUSSE L, MAURIÈGE P: Contribution of genetic and metabolic syndrome to omental adipose tissue PAI-1 gene mRNA and plasma levels in obesity. Obes Surg 20: 492-499, 2010.

CASSAR S, TEEDE HJ, HARRISON CL, JOHAM AE, MORAN LJ, STEPTO NK: Biomarkers and insulin sensitivity in women with Polycystic Ovary Syndrome: Characteristics and predictive capacity. Clin Endocrinol (Oxf) 83: 50-58, 2015.

CEDERNAES J, SCHIÖTH HB, BENEDICT C: Determinants of shortened, disrupted, and mistimed sleep and associated metabolic health consequences in healthy humans. Diabetes 64: 1073-1080, 2015.

CHEDRAUI P, PÉREZ-LÓPEZ FR, ESCOBAR GS, PALLA G, MONTT-GUEVARA M, CECCHI E, GENAZZANI AR, SIMONCINI T; RESEARCH GROUP FOR THE OMEGA WOMEN'S HEALTH PROJECT: Circulating leptin, resistin, adiponectin, visfatin, adipsin and ghrelin levels and insulin resistance in postmenopausal women with and without the metabolic syndrome. Maturitas 79: 86-90, 2014.

DEMARCO VG, SOWERS JR: Ghrelin: a new incretin enhancer therapy? Diabetes 64: 1500-1502, 2015.

FANTUZZI G, FAGGIONI R: Leptin in the regulation of immunity, inflammation, and hematopoiesis. J Leukoc Biol 68 : 437-446, 2000.

FARSHCHIAN F, RAMEZANI TF, AMIRRASOULI H, RAHIMI PH, HEDAYATI M, KAZEROUNI F, SOLTANI A: Visfatin and resistin serum levels in normal-weight and obese women with polycystic ovary syndrome. Int $J$ Endocrinol Metab 12: e15503, 2014.

FERREIRA AF, REZENDE JC, VAIKOUSI E, AKOLEKAR R, NICOLAIDES KH: Maternal serum visfatin at 11-13 weeks of gestation in gestational diabetes mellitus. Clin Chem 57: 609-613, 2011.

GHOSHAL K, BHATTACHARYYA M: Adiponectin: probe of the molecular paradigm associating diabetes and obesity. World J Diabetes 6: 151-166, 2015.

GREENMAN Y, GOLANI N, GILAD S, YARON M, LIMOR R, STERN N: Ghrelin secretion is modulated in a nutrientand gender-specific manner. Clin Endocrinol (Oxf) 60: 382-388, 2004.

GREGORASZCZUK EL, RAK A: Superactive human leptin antagonist reverses leptin-induced excessive progesterone and testosterone secretion in porcine ovarian follicles by blocking leptin receptors. J Physiol Pharmacol 66: 39-46, 2015.

HAMADA M, ABE M, MIYAKE T, KAWASAKI K, TADA F, FURUKAWA S, MATSUURA B, HIASA Y, ONJI M: $\mathrm{B}$ cell-activating factor controls the production of adipokines and induces insulin resistance. Obesity 19: 1915-1922, 2011.

HAYES AL, XU F, BABINEAU D, PATEL SR: Sleep duration and circulating adipokine levels. Sleep 34: 147-152, 2011.

KULINA GR, RAYFIELD EJ: The role of glucagon in the pathophysiology and management of diabetes. Endocr Pract 22: 612-621, 2016.

LEWANDOWSKI KC, STOJANOVIC N, PRESS M, TUCK SM, SZOSLAND K, BIENKIEWICZ M, VATISH M, LEWINSKI A, PRELEVIC GM, RANDEVA HS: Elevated serum levels of visfatin in gestational diabetes: a comparative study across various degrees of glucose tolerance. Diabetologia 50: 1033-1037, 2007.

LO JC, LJUBICIC S, LEIBIGER B, KERN M, LEIBIGER IB, MOEDE T, KELLY ME, CHATTERJEE BHOWMICK D, MURANO I, COHEN P, BANKS AS, KHANDEKAR MJ, DIETRICH A, FLIER JS, CINTI S, BLÜHER M, DANIAL NN, BERGGREN PO, SPIEGELMAN BM: Adipsin is an adipokine that improves $\beta$ cell function in diabetes. Cell 158: 41-53, 2014. 
MASLOWSKA M, SNIDERMAN AD, GERMINARIO R, CIANFLONE K: ASP stimulates glucose transport in cultured human adipocytes. Int J Obes Relat Metab Disord 21: 261-266, 1997.

MEHTA NN, MCGILLICUDDY FC, ANDERSON PD, HINKLE CC, SHAH R, PRUSCINO L, TABITA-MARTINEZ J, SELLERS KF, RICKELS MR, REILLY MP: Experimental endotoxemia induces adipose inflammation and insulin resistance in humans. Diabetes 59: 172-181, 2010.

MOSCAVITCH SD, KANG HC, FILHO RA, MESQUITA ET, NETO HC, ROSA ML: Comparison of adipokines in a cross-sectional study with healthy overweight, insulin-sensitive and healthy lean, insulin-resistant subjects, assisted by a family doctor primary care program. Diabetol Metab Syndr 8: 9, 2016.

NASRAT H, PATRA SK, GOSWAMI B, JAIN A, RAGHUNANDAN C: Study of association of leptin and insulin resistance markers in patients of PCOS. Indian J Clin Biochem 31: 104-107, 2016.

OSEGBE I, OKPARA H, AZINGE E: Relationship between serum leptin and insulin resistance among obese Nigerian women. Ann Afr Med 15: 14-19, 2016.

RASK E, OLSSON T, SÖDERBERG S, HOLST JJ, TURA A, PACINI G, AHRÉN B: Insulin secretion and incretin hormones after oral glucose in non-obese subjects with impaired glucose tolerance. Metabolism 53: 624-631, 2004.

RAVN P: New paradigms in PCOS: impaired glucose tolerance and cardiovascular risk. Clinical approach. Minerva Ginecol 67: 217-223, 2015.

RIZK NM, SHARIF E: Leptin as well as free leptin receptor is associated with polycystic ovary syndrome in young women. Int J Endocrinol 2015: 927805, 2015.

SEOW KM, JUAN CC, WU LY, HSU YP, YANG WM, TSAI YL, HWANG JL, HO LT: Serum and adipocyte resistin in polycystic ovary syndrome with insulin resistance. Hum Reprod 19: 48-53, 2004.

SEOW KM, JUAN CC, HO LT, HSU YP, LIN YH, HUANG LW, HWANG JL: Adipocyte resistin mRNA levels are down-regulated by laparoscopic ovarian electrocautery in both obese and lean women with polycystic ovary syndrome. Hum Reprod 22: 1100-1106, 2007.

SEPILIAN VP, CROCHET JR, NAGAMANI M: Serum soluble leptin receptor levels and free leptin index in women with polycystic ovary syndrome: relationship to insulin resistance and androgens. Fertil Steril 85: 1441-1447, 2006.

SPRANGER J, KROKE A, MÖHLIG M, BERGMANN MM, RISTOW M, BOEING H, PFEIFFER AF: Adiponectin and protection against type 2 diabetes mellitus. Lancet 361: 226-228, 2003.

THOMAS S, SURESH S, SUDHEESH M, VIJAYAKUMAR T: Association of insulin resistance with adipocytokine levels in patients with metabolic syndrome. Indian J Clin Biochem 30: 155-160, 2015.

VERHULST PJ, DEPOORTERE I: Ghrelin's second life: from appetite stimulator to glucose regulator. World $J$ Gastroenterol 18: 3183-3195, 2012.

VOLLMER K, HOLST JJ, BALLER B, ELLRICHMANN M, NAUCK MA, SCHMIDT WE, MEIER JJ: Predictors of incretin concentrations in subjects with normal, impaired, and diabetic glucose tolerance. Diabetes 57: 678-687, 2008.

WEI J, GAO J, CHENG J: Gestational diabetes mellitus and impaired glucose tolerance pregnant women. Pak J Med Sci 30: 1203-1208, 2014.

YADAV A, KATARIA MA, SAINI V, YADAV A: Role of leptin and adiponectin in insulin resistance. Clin Chim Acta 417: 80-84, 2013.

YASRUEL Z, CIANFLONE K, SNIDERMAN AD, ROSENBLOOM M, WALSH M, RODRIGUEZ MA: Effect of acylation stimulating protein on the triacylglycerol synthetic pathway of human adipose tissue. Lipids 26: 495-499, 1991.

ZHANG F, TANG X, CAO H, LÜ Q, LI N, LIU Y, ZHANG X, ZHANG Y, CAO M, WAN J, AN Z, TONG N: Impaired secretion of total glucagon-like peptide-1 in people with impaired fasting glucose combined impaired glucose tolerance. Int J Med Sci 9: 574-581, 2012. 